\title{
Controlled Magnetization by Electron Holography of Polycrystalline Cobalt Nanowires
}

\author{
John E. Sanchez ${ }^{1}$, Jesus Cantu-Valle ${ }^{1}$, Eduardo Ortega ${ }^{1}$, Israel Betancourt ${ }^{2}$, Mazin M. Maqableh ${ }^{3}$, \\ Bethanie J.H. Stadler ${ }^{3}$ Miguel Jose Yacaman ${ }^{1}$ and Arturo Ponce ${ }^{1}$ \\ 1. Department of Physics and Astronomy, University of Texas at San Antonio. One UTSA Circle, San \\ Antonio, TX, USA. \\ 2. Instituto de Investigaciones en Materiales, Universidad Nacional Autónoma de México, C.P., México. \\ 3. Electrical and Computer Engineering, University of Minnesota, 4-174 EE/CSci Bldg., 200 Union St. \\ SE, Minneapolis, Minnesota, USA
}

Nowadays the comprehensive understanding of nanoscale materials and their physical properties are of great interest to the scientific and technological community. In particular, magnetic nanostructures of different size, shape and composition (e.g. nanoparticles, nanowires or thin films) possess a great potential to improve current technologies in areas such as: magnetic data storage, electromagnetic sensing [1-2]. Lately, soft magnetic nanowires, (Co, Fe \& $\mathrm{Ni}$ ) have been studied for a while experimentally and by simulations, but there still some questions to be address. Soft magnetic nanowires can switch magnetization in two different modes depending on their thickness, these modes are known as the transverse wall mode and the vortex wall mode. In thin ferromagnetic nanowires (diameter less than 40nm) a simple domain wall nucleates and propagates along the nanowire axis, while the reversal of thick nanowires (diameter more than $40 \mathrm{~nm}$ ) is achieved via localized curling or vortex mode. The magnetization direction of each magnetic domain will be influenced by the magnetocrystalline anisotropy; typically following the easy magnetization axis, which minimize the magnetocrystalline energy. The magnetization behavior in this nanostructures is dominated by the competition between magnetocrystalline anisotropy and shape anisotropy. In many cases this competition between can frustrates the magnetization direction. It is expected that the magnetostatic coupling between nanostructures have a strong influence on their response to an external field [3].

In this paper we report in situ TEM magnetization distribution of cobalt nanowires as a function of variable magnetic field produced by the systematic control of the objective lens voltage in a JEOL ARM200F microscope. A modified holder coupled with a Hall sensor was implemented to monitor the intensity of the magnetic field. Off-axis electron holography under Lorentz conditions was used for mapping the magnetic flux of individual cobalt nanowires in their initial configuration. Subsequently, we show magnetization reorientations and magnetization reversals for isolated nanowires by changing the excitation voltage of the objective lens, which produces variable magnetic field along the nanowire main axis. The formation of complex magnetic configurations are discussed in terms of local competition among variable anisotropy easy axis directions afforded by misaligned grains.

Cobalt nanowires were fabricated using an anodic aluminum oxide (AAO) template with columnar nanopores of $95 \pm 5 \mathrm{~nm}$ diameter by electrochemical deposition. The microscope is equipped with a pole piece model UHR22, which possesses a remanent field of 45 Oe. The magnetic contribution of the objective lens excitation was measured with a Hall effect sensor adapted in a Nanofactory Instruments Inc. holder within the column of the microscope. The sensor used (model HSU-1 from Cryomagnetics Inc.) for the measurement of the magnetic field has an active area of $0.1 \times 0.1$ [mm] with a sensitivity > $5[\mathrm{mV} / \mathrm{T}]$ and it was welded to the Nanofactory chip channels in order to avoid additional wiring. These 
welding points were cover with an adhesive (GE-7031) made by General Electric to provide electrical insulation. Then the sensor was connected to a Gaussmeter model GM-700 as shown in Figure 1a. Subsequently, the chip is mounted in the holder, thus the cap of the holder provides the electrical contacts (Figure 1b). Finally the holder outputs were connected directly to the Gaussmeter to register the field measurements as shown in Figure 1c. The complete experimental setup can be visualized in Figure 1d. The effect of the increment of the external magnetic field up to $1.6 \mathrm{~T}$ along the nanowire's longitudinal main axis (corresponding to an OL voltage of 6.0 volts) is displayed in Figure 2. The component $\mathrm{Hp}$ is now of $0.54 \mathrm{~T}$, which produces a marked reorientation of the magnetization along the axial direction, even at the tip, for which the decomposition of the vortex is almost completed. The symmetry of the external magnetic flux lines suggests that once the vortex is reduced to a minimum, the local variations of the c-axis of disoriented grains located at the tip prevail over the magnetizing effect of Hp, making the role of a domain wall, hence facilitating the symmetric leak of the magnetic lines, in a similar way to the observed for head-to-head magnetic domain walls in equivalent magnetic nanowires [4]. A predominant magnetization orientation along the longitudinal nanowire axis results from the prevailing influence of the shape anisotropy, but at the nanowire tip, local magnetocrystalline anisotropy variations, afforded by disoriented grains, allow the formation of vortex structures with surrounding uncompensated magnetic flux, yielding to asymmetric stray field configuration out of the nanowires.

\section{References:}

[1] V.F. Puentes et al, Science 291 (2001) 2115.

[2] T.M. Whitney et al, Science 261, (1993) 1316.

[3] G. Bergmann, et al, Phys. Rev. B 77 (2008) 054415.

[4] N.Biziere, et al, Nano Lett. 13 (2013) 2053.
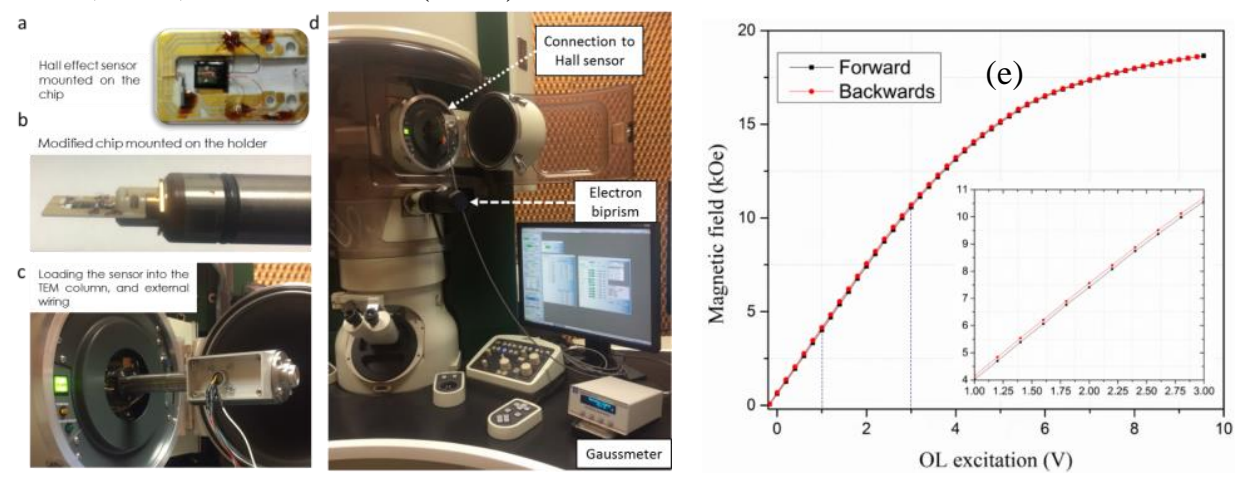

Figure 1. (a) Hall effect sensor welded to the commercial sensor. (b) Chip mounted in the holder, the cap of the holder provides the electrical contacts (c) wiring the holder outputs through the Gaussmeter (d) Picture of the mounted experimental setup in the microscope. (e) Magnetic field at the specimen position as a function of the objective lens (OL) excitation voltage. Black curve is the forward bias, and red is the backwards, showing no significant hysteresis. An expansion of the linear part of the curve (inset) shows that there is only a minor hysteresis in the lens.

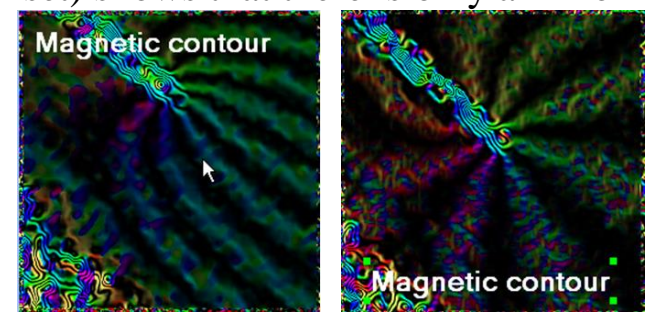

Figure 2. Magnetic contours of the Co nanowire under the effect of an external magnetic field of (left) 1.25 Tesla and (right) 1.6 Tesla. 